\title{
A INFLUÊNCIA DOS CONTEXTOS SOCIODEMOGRÁFICOS NO BONDING PATERNO
}

\author{
Carla Cruz \\ Paula Nelas \\ Emília Coutinho \\ Cláudia Chaves \\ Odete Amaral \\ Escola Superior de Saúde de Viseu \\ Instituto Politécnico de Viseu - CI\&DETS \\ cruzcarla@hotmail.com \\ https://doi.org/10.17060/ijodaep.2017.n1.v3.980
}

Fecha de Recepción: 26 Enero 2017

Fecha de Admisión: 1 Abril 2017

\section{RESUMO}

Enquadramento: 0 processo de vinculação é indispensável pois favorece e/ou determina a futura relação entre a díade: pai/bebé e influencia o desenvolvimento cognitivo e social da criança. Este processo de vinculação (bonding) é condicionado por um conjunto de fatores interligados com as condições parentais e situacionais que poderão coadjuvar ou impedir o estabelecimento do processo de vinculação (Pereira, 2009).

Objetivo: Estabelecer a relação entre o contexto sociodemográfico (idade, estado civil, habilitações literárias e duração do relacionamento) e a vinculação (bonding) entre o pai e 0 bebé.

Método: Trata-se de um estudo transversal, descritivo-correlacional, de natureza quantitativa, com uma amostra de 349 pais com média de idade de 31,84 anos $(\mathrm{dp}=6,067)$. 0 instrumento de colheita de dados foi 0 questionário que permitiu caracterizar a amostra nas variáveis sociodemográficas e a Escala de Bonding (Figueiredo et al., 2005).

Resultados: Os resultados demonstram que 0 bonding positivo é influenciado pela idade $(p=0,010)$, sendo mais elevado nos pais com idade igual ou inferior a 30 anos. No que concerne às habilitações literárias, verificou-se que quanto menor é a escolaridade dos pais, maior é o bonding positivo $(p=0,045)$.Por outro lado o estado civil e a duração do relacionamento não influenciam 0 bonding.

Conclusão: Face à importância do processo de vinculação pai/bebe, cujo envolvimento deve existir desde o início da gravidez, é imprescindível que o Enfermeiro especialista em saúde materna assuma a responsabilidade de educar e estimular o bonding paterno apoiando e integrando o pai no processo de adaptação à parentalidade.

Palavras-chave: Pai; Bebé; Bonding; gravidez 


\section{A INFLUÊNCIA DOS CONTEXTOS SOCIODEMOGRÁFICOS NO BONDING PATERNO}

\section{ABSTRACT}

\section{The influence of sociodemographic context on parent/baby bonding}

Background: The bonding process is essential to favors and/or determines the future relationship between parent and baby and influences the cognitive and social development of the child. The bonding is conditioned by a set of interrelated factors with parental and situational conditions that may assist or prevent the establishment of bonding (Pereira, 2009).

Objective: Establish the relationship between the sociodemographic context (age, marital status, educational qualifications and length of relationship) and bonding parent/baby.

Methods: This study is a cross-sectional study, correlational descriptive, quantitative, attended by 349 parents, aged between 19 and 55, with an average age of 31,84 ( $s d=6,067$ ). To providing a reliable sample characterization the social-demographic we applied questionnaires and Bonding Scale (Figueiredo et al., 2005).

Results: This study reveals that positive bonding is influenced by age $(p=0,010)$, being higher in parents aged 30 years or less. With regard to qualifications, it was found that the smaller the educational level of the parents, the greater the bonding positive $(p=0,045)$. On the other hand the marital status and the length of the relationship did not influence the bonding.

Conclusion: Given the importance of the bonding process between parent and baby, whose involvement should exist from the beginning of pregnancy, it s imperative that the Nurse Specialist in maternal health take responsibility to educate and stimulate the paternal bonding supporting and integrating the father in the process of adaptation to parenthood.

Keywords: Father, Baby, Bonding; Pregnancy

\section{INTRODUÇÃO}

0 nascimento de um bebé é um acontecimento relevante que provoca mudanças e adaptações na vida do casal e no ciclo de vida familiar, alterando os comportamentos, as relações e as funções de pais e mães, envolvendo modificações de perspetivas, reorganização da família e um aumento de responsabilidades (PALÁCIOS, 2005) dando lugar a uma sequência previsível de transformações na organização familiar (RELVAS, 2004).

0 pai necessita de orientação e apoio para aprender a viver o processo de transição para a parentalidade que envolve novas experiencias de afeto e sentimentos de vínculo que englobam todos os comportamentos que Ihe irão permitir estabelecer e manter a proximidade ou contacto com o bebé (BRANDÃO, 2009).

0 processo de vinculação (bonding) é condicionado por um conjunto de fatores interligados com as condições parentais e situacionais que poderão coadjuvar ou impedir o estabelecimento do vínculo (PEREIRA, 2009), indispensável, favorecedor e/ou determinante da relação pai/bebé e influenciador do desenvolvimento cognitivo e social da criança.

A interação estabelecida entre 0 pai e 0 bebé promove 0 bonding, um processo gradativo de envolvimento afetivo entre esta díade. Este é um processo que decorre durante o período de gravidez, nos primeiros momentos após o nascimento do bebé e no pós-parto. Figueiredo et al (2005) salientam que o bonding não ocorre imediatamente após o nascimento, ou seja, é um processo que se declara e fortalece progressivamente no decorrer do primeiro ano de vida. Para estes autores, a formação do vínculo entre pai e filho costuma ser mais lenta do que entre mãe e filho, consolidando-se gradualmente após o nascimento e no decorrer do desenvolvimento da criança.

0 pai ao assistir ao nascimento do filho vive experiências positivas pelo suporte emocional que proporciona à companheira/mulher e pelos sentimentos e emoções que experiencia, os quais podem favorecer o maior envolvimento emocional precoce com o filho (CARVALHO, 2003). 
Contudo, e tendo em conta o tema deste trabalho, o bonding poderá ser influenciado por contextos sociodemográficos, nomeadamente a idade, estado civil, habilitações literárias e duração do relacionamento.

A consciência desta realidade, consubstanciada com a motivação pessoal, aleada às evidências de poucos estudos que se enfoquem no estabelecimento de laços afetivos entre o pai e o bebé no trabalho de parto e parto, levou à elaboração da seguinte questão central, subjacente à problemática do presente estudo: Em que medida as variáveis sociodemográficas influenciam o bonding?

$\mathrm{Na}$ atualidade as evidências mostram que o pai quer ser cada vez mais um elemento ativo no momento do parto, considerando fulcral a sua participação enquanto companheiro que dá apoio físico e emocional à mãe. Este experiencia sentimentos positivos e negativos nem sempre vão ao encontro ao idealizado, podendo sofrer influência de vários fatores.

Assim, propomos-mos alcançar o seguinte objetivo: Estabelecer a relação entre o contexto sociodemográfico (idade, estado civil, habilitações literárias e duração do relacionamento) e a vinculação (bonding) entre o pai e o bebé.

Consideramos esta problemática muito atual e pertinente, esperando que este trabalho, desenvolva 0 sentido crítico, sobre o bonding aleado à figura paterna, servindo de ponto de partida para 0 desenvolvimento de linhas orientadoras da intervenção dos enfermeiros especialmente que lidam diariamente com as questões da natalidade.

\section{MATERIAL E MÉTODOS}

Optámos por realizar um estudo quantitativo, do tipo transversal, descritivo-correlacional, com uma amostra intencional não probabilística por conveniência, constituída por 349 progenitores do sexo masculino, com idade compreendida entre os 19 aos 55 anos, ( $x=31,84$ anos) e ( $d p=6,067)$. A maioria tem com habilitações literárias até ao 3 ํㅜ Ciclo (37,4\%), são casados $(66,9 \%)$ e mantêm uma relação conjugal entre os 4 e os 7 anos (35,8\%).

0 bonding (processo de envolvimento afetivo entre o pai e o bebé) é a variável dependente e as variáveis sociodemográficas a variável independente.

0 instrumento de colheita de dados é composto por um questionário de caracterização sociodemográfica do pai, constando de 5 questões, nomeadamente: idade, raça, habilitações literárias, estado civil e duração de relacionamento com a parturiente e a Escala de Bonding de Figueiredo et al em 2005, que irá permitir avaliar o envolvimento emocional dos pais com o bebé. Esta escala está dividida em três subescalas que avaliam o Bonding positivo, Bonding negativo e o Bonding not clear. 0 Bonding Total, que é obtido através da subtração das subescalas "bonding negativo" e "bonding Not Clear" à subescala "bonding Positivo".

No decurso do processo de colheita de dados foi garantida a confidencialidade e a privacidade, assim como obtido o consentimento informado do respondente para a participação no estudo, pelo preenchimento livre e esclarecido do questionário.

\section{RESULTADOS}

Os resultados demonstram que 0 bonding positivo é influenciado pela idade $(p=0,010)$, sendo mais elevado nos pais com idade igual ou inferior a 30 anos. No que concerne às habilitações literárias, verificou-se que quanto menor é a escolaridade dos pais, maior é o bonding positivo $(p=0,045)$. Por outro lado concluímos que 0 estado civil e a duração do relacionamento não influenciam 0 bonding.

\section{DISCUSSÃO}

Diversos autores estudaram a relação entre fatores sociodemográficos e 0 bonding paterno. 


\section{A INFLUÊNCIA DOS CONTEXTOS SOCIODEMOGRÁFICOS NO BONDING PATERNO}

Também foi nossa preocupação perceber a relação entre o contexto sociodemográfico (idade, estado civil, habilitações literárias e duração do relacionamento) e a vinculação (bonding) entre o pai e 0 bebé.

\section{RELAÇÃO ENTRE A IDADE E 0 BONDING}

Os resultados apurados revelaram que no bonding positivo, no bonding not clear e no bonding total, as médias são mais elevadas nos sujeitos com idade igual ou inferior a 30 anos. No bonding negativo, as médias são mais elevadas nos participantes com idade igual ou superior a 31 anos. Ao realizarmos o cruzamento entre a idade e as diversas subescalas do bonding, verificámos que existem diferenças estatisticamente significativas entre 0 bonding positivo $(p=0,010)$ e a idade (cf. Quadro 1).

Desta forma podemos referir que a idade influencia o bonding positivo. Estes resultados confirmam a ideia de Parke (1996) Cit. por Boli (2002) que refere que o período da vida em que o individuo se torna pai é muito importante já que a paternidade na adolescência é distinta da paternidade vivenciada num homem em idade adulta. Também Lima (2006) e Saraceno e Naldini (2003) Cit. por Ferreira (2010), referem que os pais mais jovens têm uma maior reivindicação na relação com os filhos. Segundo Magalhães, Patinha e Conceição (2003) Cit. por Mendes (2007) a idade é um factor predisponente para os pais assistirem ao parto. Pelo contrário, um estudo de Ferreira (2010) sobre 0 envolvimento do pai na gravidez revelou que não existe correlação entre 0 envolvimento paterno e a idade.

Quadro 1

Teste $t$ entre a idade e o bonding

\begin{tabular}{|l|c|c|c|c|c|c|}
\hline \multirow{2}{*}{ Bonding } & \multicolumn{2}{|c|}{$\begin{array}{c}\text { Inferior ou igual a 30 } \\
\text { anos }\end{array}$} & \multicolumn{2}{c|}{$\begin{array}{c}\text { Superior ou igual a 31 } \\
\text { anos }\end{array}$} & \multirow{2}{*}{$\mathrm{T}$} \\
\cline { 2 - 7 } & Média & DP & Média & DP & & \\
\hline Bonding negativo & 0,23 & 0,96 & 0,31 & 0,87 & $-0,742$ & 0,459 \\
\hline Bonding positivo & 8,44 & 1,08 & 8,12 & 1,19 & 2,598 & 0,010 \\
\hline Bonding not clear & 1,50 & 1,38 & 1,45 & 1,25 & 0,396 & 0,692 \\
\hline Bonding total & 6,70 & 2,21 & 6,36 & 2,03 & 1,440 & 0,151 \\
\hline
\end{tabular}

\section{RELAÇÃO ENTRE AS HABILITAÇÕES LITERÁRIAS E 0 BONDING}

Confirmamos que, na amostra em estudo, tanto no bonding negativo, como no positivo e no total, as médias mais elevadas centraram-se nos participantes com escolaridade até ao 3ํㅜ ciclo e as médias mais baixas nos participantes com 0 ensino superior. Contudo, no bonding not clear, a média mais elevada foi encontrada nos pais com ensino superior e a média mais baixas nos que possuíam habilitações até ao $3^{\circ}$ ciclo. Foram encontradas diferenças estatisticamente significativas $(p=0,045)$ apenas para 0 bonding positivo (cf. Quadro 2) pelo que podemos referir que as habilitações literárias têm influência no bonding positivo. Estudos realizados por Moura-Ramos e Canavarro (2007) referem que a adaptação à parentalidade depende do contexto em que 0 individuo se insere, nomeadamente nos papéis sociais que desempenha. Também Ribeiro (2005) Cit. por Felizardo (2010) refere que o nível socioeconómico e cultural interferem no desejo do pai em assistir ao parto. 
Quadro 2

Teste One-Way ANOVA entre as habilitações literárias e o Bonding

\begin{tabular}{|c|c|c|c|c|c|c|c|}
\hline Bonding & Habilitações Literárias & $\mathrm{N}$ & Média & DP & $\mathrm{F}$ & $\mathrm{P}$ & Tukey \\
\hline \multirow{3}{*}{$\begin{array}{l}\text { Bonding } \\
\text { Negativo }\end{array}$} & Até ao $3^{\circ}$ Ciclo & 130 & 0,38 & 1,16 & \multirow[t]{3}{*}{1,603} & \multirow[t]{3}{*}{0,203} & - \\
\hline & Ensino Secundário & 129 & 0,23 & 0,72 & & & - \\
\hline & Ensino Superior & 89 & 0,17 & 0,66 & & & - \\
\hline \multirow{3}{*}{$\begin{array}{l}\text { Bonding } \\
\text { Positivo }\end{array}$} & Até ao $3^{\circ}$ Ciclo & 130 & 8,43 & 1,06 & \multirow[t]{3}{*}{3,133} & \multirow[t]{3}{*}{0,045} & 0,040 \\
\hline & Ensino Secundário & 129 & 8,20 & 1,19 & & & - \\
\hline & Ensino Superior & 89 & 8,04 & 1,22 & & & - \\
\hline \multirow{3}{*}{$\begin{array}{l}\text { Bonding } \\
\text { Not Clear }\end{array}$} & Até ao $3^{\circ}$ Ciclo & 130 & 1,34 & 1,43 & \multirow[t]{3}{*}{1,528} & \multirow[t]{3}{*}{0,218} & - \\
\hline & Ensino Secundário & 129 & 1,47 & 1,26 & & & - \\
\hline & Ensino Superior & 89 & 1,65 & 1,15 & & & - \\
\hline \multirow{3}{*}{$\begin{array}{l}\text { Bonding } \\
\text { Total }\end{array}$} & Até ao $3^{\circ}$ Ciclo & 130 & 6,71 & 1,43 & \multirow[t]{3}{*}{1,436} & \multirow[t]{3}{*}{0,239} & - \\
\hline & Ensino Secundário & 129 & 6,50 & 1,27 & & & - \\
\hline & Ensino Superior & 89 & 6,22 & 1,15 & & & - \\
\hline
\end{tabular}

\section{RELAÇÃO ENTRE 0 ESTADO CIVIL E 0 BONDING}

Os pais casados apresentam níveis mais elevados de bonding negativo, comparativamente com os participantes que vivem em união de facto. Os casados apresentam níveis médios mais elevados de bonding positivo, comparativamente aos solteiros. Por último, os pais que vivem em união de facto apresentam níveis mais elevados de bonding not clear comparativamente com os pais solteiros. No bonding total, apuramos que são os solteiros que apresentam médias mais elevadas, comparativamente com os que vivem em união de facto. Contudo, não existem diferenças estatisticamente significativas, isto é, o estado civil não interfere nas diferentes subescalas de bonding. 0 facto de terem sido os pais casados a ter um bonding positivo poderá estar relacionado com a própria transição para a parentalidade que é pautada pela mudança a diversos níveis, exigindo um processo de adaptação no qual importa cumprir um conjunto de tarefas (CONDE E FIGUEIRED0, 2007). As transições resultam em mudanças na vida do casal, nos seus relacionamentos e no ambiente. Assim, os pais casados poderão já ter passado por estas adaptações e, como tal, estar mais propensos à vinculação afetiva com o recém-nascido. Há a considerar também que a maioria dos participantes deste estudo é casada (66,9\%). Estes resultados demonstram que as características individuais dos pais, especialmente a relação conjugal, são fundamentais para 0 tipo de envolvimento entre 0 pai e o bebé (Levy-shiff, 1994, Cit. por Brandão, 2009). A autora citada acrescenta que a forma como o pai e a mãe vivenciam os sentimentos únicos e especiais face ao nascimento de um filho é reflexo da sua relação enquanto casal. Figueiredo et al (2008) Cit. por Brandão (2009) afirmam que os relacionamentos são muito importantes e quando se desenvolvem positivamente facilitam a transição para a parentalidade.

Quadro 3

Teste Kruskal-Wallis entre o estado civil e o bonding

\begin{tabular}{|l|c|c|c|c|c|}
\hline \multirow{2}{*}{ Bonding } & Solteiro & Casado & $\begin{array}{c}\text { União de } \\
\text { Facto }\end{array}$ & \multirow{2}{*}{$\mathrm{X}^{2}$} & $\mathrm{p}$ \\
\cline { 2 - 5 } & & $\mathrm{M}$ & $\mathrm{M}$ & $\mathrm{M}$ & \\
\hline Bonding Negativo & 175,30 & 175,44 & 165,25 & 1,179 & 0,555 \\
\hline Bonding Positivo & 170,27 & 175,11 & 173,82 & 0,161 & 0,923 \\
\hline Bonding Not Clear & 162,28 & 173,95 & 190,58 & 2,383 & 0,304 \\
\hline Bonding Total & 182,48 & 175,02 & 157,26 & 1,896 & 0,388 \\
\hline
\end{tabular}




\section{A INFLUÊNCIA DOS CONTEXTOS SOCIODEMOGRÁFICOS NO BONDING PATERNO}

\section{RELAÇÃO ENTRE A DURAÇÃO DA RELAÇÃO E 0 BONDING}

Os pais que estão na relação há 8 ou mais anos possuem níveis mais elevados de bonding negativo e not clear. Os participantes que estão na relação atual há menos tempo (0-3 anos) possuem níveis mais elevados de bonding positivo. Todavia, os valores médios não resultaram em diferenças estatisticamente significativas pelo que constatamos que a duração da relação tem pouco impacto no bonding (cf. Quadro 3). Neste âmbito, Genesosi (2009); Ribeiro (2005) Cit. por Felizardo (2010), bem como Parke (1996) Cit. por Castoldi (2002), afirmam que a qualidade do relacionamento do casal influencia a vontade do pai em assistir ao nascimento e potencia o envolvimento emocional deste com o seu bebé. Também, Ramos e Cecílio (1997) Cit. por Brandão (2009), referem que ser pai resulta da aceitação de uma relação mais ou menos permanente com a mãe do bebé e a vontade do pai em se envolver está relacionada com o tipo de relação que existe entre 0 casal.

Quadro 4

Teste One-Way ANOVA entre a duração da relação e o bonding

\begin{tabular}{|c|c|c|c|c|c|c|c|c|}
\hline \multirow{2}{*}{$\begin{array}{l}\text { Duração da relação } \\
\text { atual }\end{array}$} & \multicolumn{2}{|c|}{ Entre 0 a 3 anos } & \multicolumn{2}{|c|}{ Entre 4 a 7 anos } & \multicolumn{2}{|c|}{8 ou mais anos } & \multirow[t]{2}{*}{$\mathrm{F}$} & \multirow[t]{2}{*}{$\mathrm{p}$} \\
\hline & $\mathbf{M}$ & DP & $\mathbf{M}$ & DP & $\mathbf{M}$ & DP & & \\
\hline Bonding Negativo & 0,14 & 0,40 & 0,33 & 1,08 & 0,34 & 1,01 & 1,740 & 0,177 \\
\hline Bonding Positivo & 8,32 & 1,09 & 8,24 & 1,18 & 8,20 & 1,19 & 0,339 & 0,713 \\
\hline Bonding Not Clear & 1,36 & 1,29 & 1,47 & 1,33 & 1,56 & 1,28 & 0,619 & 0,539 \\
\hline Bonding Total & 6,82 & 1,71 & 6,44 & 2,34 & 6,29 & 2,16 & 1,831 & 0,162 \\
\hline
\end{tabular}

\section{CONCLUSÕES}

Constatamos com base na revisão de literatura, que a vinculação paterna é um processo de envolvimento afetivo do pai com o bebé, que se constrói gradualmente com o desenvolvimento de determinadas competências.

É neste contexto que suportamos teoricamente o objetivo geral deste trabalho, o qual consistiu em estudar a relação entre os contextos sociodemográficos e o bonding na relação pai/bebé.

Pretendemos que esta investigação contribua com dados relevantes para a prática do cuidar permitindo-nos identificar algumas relações estatisticamente significativas das diferentes variáveis com o bonding e responder á questão central do estudo:

Em que medida as variáveis sociodemográficas (idade, estado civil, habilitações literárias e duração do relacionamento) influenciam o bonding?

A análise e discussão dos dados deste estudo empírico mostraram que da relação entre as variáveis sociodemográficas e o bonding, a idade e as habilitações literárias o influenciam de forma significativa. Inferimos, então que a idade apenas interfere no bonding positivo $(p=0,010)$, sendo que este é mais elevado nos pais com idade igual ou inferior a 30 anos. No que concerne às habilitações literárias, verificou-se que quanto menor é a escolaridade dos pais, maior é o bonding positivo $(p=0,045)$.

Os enfermeiros devem olhar o pai de forma holística, atendendo ao contexto em que este está inserido, disponibilizando-se para ajudar e esclarecer dúvidas já que os pais referem que o apoio dos profissionais de saúde, no ciclo gravídico e parto, é fulcral para que se desenvolva uma relação precoce e positiva entre a tríade pai/mãe/filho.

Dado o facto de os enfermeiros especialistas em saúde materna serem privilegiados no que diz respeito ao acompanhamento do casal gravídico durante todo o processo de gravidez, trabalho de parto e parto, é importante que estes compreendam e interpretem os sentimentos expectantes do pai e planeiem os cuidados, envolvendo-o neste processo, atendendo às suas necessidades.

0 conhecimento destes dados torna-se relevante para que 0 enfermeiro possa basear as suas 
práticas em evidências científicas e desenvolver competências que contribuam para a melhoria contínua dos cuidados prestados.

\section{REFERÊNCIAS BIBLIOGRÁFICAS}

Bolli, A. C. B. (2002). 0 envolvimento paterno com o bebé e aos doze meses de idade (Dissertação de mestrado, Universidade do Rio Grande do Sul). Acedido em https://www.lume.ufrgs.br/bitstream/handle/10183/2553/000372062.pdf?sequence $=1$

Brandão, S. M. P. A. (2009). Envolvimento emocional do pai com o bebé: Impacto da experiência do parto (Dissertação de Mestrado, Universidade do Porto). Acedido em https://repositorio-aberto.up.pt/handle/10216/16151

Carvalho, M. L. M. (2003). Participação dos pais no nascimento em maternidade pública: dificuldades institucionais e motivações dos casais. Cadernos Saúde Pública, (19) Sup. 2, 389-398.

Castoldi, L. (2002). A construção da paternidade desde a gestação até ao primeiro ano do bebé (Dissertação de mestrado, Universidade do Rio Grande do Sul). Acedido em https://www.lume.ufrgs.br/bitstream/handle/10183/1576/000351993.pdf?sequence $=1$

Conde, A., \& Figueiredo, B. (2007). Preocupações de mães e pais, na gravidez, parto e pós parto. Análise Psicológica, (25) 3, 381-398.

Felizardo, S. M. A. S. (2010). Os efeitos do suporte social em famílias de crianças com deficiência. In C. Nogueira, I. Silva, L. Lima, A.T. Almeida, R. Cabecinhas, R. Gomes,... M.C. Taveira (Eds), Actas do VII Simpósio Nacional de Investigação em Psicologia (pp. 1-11). Viseu: Escola Superior de Educação de Viseu, I. P. V. Acedido em http://repositorio.ipv.pt/handle/10400.19/524

Ferreira, L. S., L., Magda P., \& Néné, M. (2010). Envolvimento paterno e o planeamento da gravidez. Revista da Ciências da Saúde da ESSCVP, 2, 22-30.

Figueiredo, B., Costa, R., \& Pacheco, A. (2005). Bonding: Escala para avaliar o envolvimento emocional dos pais com o bebé. Psychologica, 40, 133-154.

Freitas, I. (2009). As relações entre suporte social e balanço emocional (Dissertação de mestrado, Universidade Metodista de São Paulo). Acedido em http://bdtd.ibict.br/vufind/Record/MET0_3da41501057cd66aca81156f966d8628/Details

Mendes, R. V. (2007). A parentalidade experimentada no masculino: As vivências da paternidade. CIES e-Working Paper, 22, 1-24. Acedido em https://repositorio.iscte-iul.pt/handle/10071/285? locale=en

Moura-Ramos, M., \& Canavarro, M. C. (2007). Adaptação parental ao nascimento de um filho: comparação da reactividade emocional e psicossintomatologia entre pais e mães nos primeiros dias após o parto e oito meses após o parto. Análise Psicológica, (25)3, 399-413. Acedido em http://repositorio.ispa.pt/handle/10400.12/109

Pereira, M. A. R. A. (2009). 0 primeiro contacto pai-bebé: um olhar sobre a prática (Dissertação de Mestrado, Universidade do Porto). Acedido em https://repositorio-aberto.up.pt/handle/10216/20151

Relvas, A. P. (2004). 0 ciclo vital da família, perspectiva sistémica. Porto: Editora Afrontamento. 
